\title{
VALIDAÇÃO E REPRODUTIBILIDADE DE PLATAFORMA DE SALTO COM LASER PARA ALTURA DO SALTO EM VOLEIBOLISTAS
}

Leonardo dos Santos Oliveira

Solon José Gonçalves de Sousa

Richard Valadares Silva

Pedro Ianaí Pordeus Uchoa

Péricles Paes Barreto Correia

Idico Luiz Pellegrinotti

Gilmário Ricarte Batista

\section{Resumo}

A proposta deste estudo é analisar a validade e a reprodutibilidade de uma plataforma de salto com laser para medir a altura do salto em voleibolistas. A amostra foi formada por 18 praticantes de voleibol da cidade de Campina Grande/PB. Os indivíduos realizaram uma seqüência de 5 bloqueios com 15 segundos de intervalo na zona de salto ao centro da rede. Utilizou-se médias e coeficiente de variação, medida Alpha de Cronbach, correlação de Pearson e coeficiente de correlação intraclasse (CCI) para repetibilidade e reprodutibilidade. Todas as correlações encontradas foram acima de 0,60 e os CCI de 0,90 , indicando que o processo de validação é pertinente. Neste estudo a proposta de validação é aceitável e satisfatória com base e de conformidade com os índices da literatura.

\section{Palavras-Chave}

Testes; Voleibol; Medidas e avaliação; Salto vertical.

\section{VALIDAÇÃO E REPRODUTIBILIDADE DE PLATAFORMA DE SALTO COM LASER PARA ALTURA DO SALTO EM VOLEIBOLISTAS}

Leonardo dos Santos Oliveira

Solon José Gonçalves de Sousa

Richard Valadares Silva

Pedro Ianaí Pordeus Uchôa

Péricles Paes Barreto Correia

Idico Luiz Pellegrinotti

Gilmário Ricarte Batista

\begin{abstract}
The purpose of this study is to analyze the validity and reproducibility of a jump platform with laser to measure the height of the jump in volleyball players. The sample was formed by 18 apprentices of volleyball of Campina Grande/PB. The individuals accomplished a sequence of 5 blocks with 15 seconds of rest period in the jump zone to the center of the net. Used averages and variation coefficient, measured Alpha of Cronbach, Pearson's Product Momentand, Intraclass correlation coefficient (ICC) for repeatibility and reproducibility. All the found correlations were above 0,60 and ICC 0,90 , indicating that the validation process is pertinent. In this study the validation purpose is acceptable and satisfactory with base and of conformity with the indexes of the literature.
\end{abstract}

\section{Key-Words}

Tests; Volleyball; Measurement and assessment; Vertical jump.

CONEXÕES, revista da Faculdade de Educação Física da UNICAMP, Campinas, v. 6, ed. especial, p. 111- , jul. 2008. 


\section{INTRODUÇÃO}

A pesquisa tecnológica aplicada aos desportos e seus benefícios ao monitoramento do processo de treinamento têm trazido grandes avanços. Sousa e Pellegrinotti (2000) em seus estudos afirmaram que no voleibol o salto vertical tem uma importância fundamental para um melhor desempenho do atleta, pois é usado para bloquear, atacar, levantar e sacar.

A utilização do salto vertical (SV) com o objetivo de monitorar ou avaliar as respostas do treinamento (intervenção) é bastante difundida na literatura. Cronin e Hansen (2005) afirmam que a medida da performance no salto vertical é uma forma bastante comum para a avaliação da força e potência. Os autores ressaltam as diversas derivações do SV com o objetivo de se aferir "qualidades" neuromusculares e de performance distintas de um indivíduo.

Apesar da ampla utilização, aceitação e importância destes diversos tipos de saltos como marcadores de performance, dados sobre a reprodutibilidade determinada através de amostras representativas são ainda limitadas. Entre estes estudos, vale destacar a investigação de Arteaga et al. (2000), na qual, se utilizou de um tapete de contato e reportou valores de reprodutibilidade (coeficiente de variação) de 6.3\% para o salto contramovimento no tocante a variabilidade dia-para-dia (estabilidade). Cronin, Hing e Mcnair (2004) avaliaram um salto com uma perna (unilateral), no tapete de contato e apresentaram coeficiente de variação de $4.5 \%$ e reprodutibilidade relativa (coeficiente intraclasse) de 0.982 entre duas sessões, separadas, aproximadamente, por sete dias. No tocante á estabilidade da performance, Hopkins (2000) afirma que a consistência da performance em um teste é relacionada à reprodutibilidade das medidas sobre múltiplas repetições. Um teste consistente e fidedigno apresenta pequena variação intra-indivíduo e alta correlação para teste-reteste.

A reprodutibilidade provê indicação do grau de precisão associado com uma medida particular e é um elemento vital na avaliação fisiológica dos atletas. Atkinson e Nevill (1998) salientam que é extremamente importante assegurar que uma medida que faz parte de uma pesquisa ou que sustente e controle o trabalho de um atleta, seja adequadamente reprodutiva e válida. Conseqüentemente, a avaliação da consistência e reprodutibilidade para os testes motores no âmbito do controle do treinamento para atletas de alto rendimento se faz fundamental.

As mensurações aplicadas no meio esportivo devem ser extremamente semelhantes às solicitações reais da atividade, não apenas em termos de tarefas específicas, mas também de maneira que reflita de 
fato a intensidade, a duração e o ritmo do trabalho (PLATONOV, 2004). Desse modo, tais testes muitas vezes, exigem atletas com um certo tempo de treinamento refletindo-se na qualidade técnica (ações motoras mais eficazes), cujas qualidades de ritmo e coordenação já estejam bem apuradas.

\section{OBJETIVOS}

O objetivo deste estudo é validar equipamento de plataforma de salto com laser e analisar a reprodutibilidade da altura do salto em voleibolistas na zona de ataque.

\section{METODOLOGIA SUJEITOS}

$A$ amostra foi composta por 18 de atletas de voleibol do sexo masculino, da categoria infanto juvenil participantes de clubes da Cidade de Campina Grande filiados a Federação Paraibana de Voleibol (FPV) e participantes dos eventos "Campeonato Estadual Infanto-Juvenil" e "Jogos Regionais e Abertos do Interior do Estado da Paraíba". Os 18 atletas possuíam idades compreendidas entre 15 e 17 anos, com

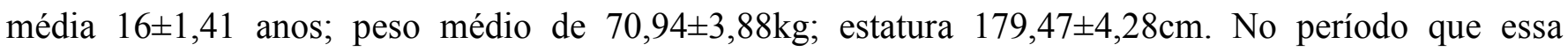
pesquisa foi realizada, os atletas se encontravam em fase de preparação física básica, ou seja, na fase inicial do treinamento. Dos 18 atletas, $58 \%$ (dez atletas) praticavam voleibol a mais de cinco anos e 48 \% (oito atletas), há mais de dois anos. Como esperado, nenhum dos indivíduos atuava como profissional.

\section{MATERIAL}

Foram utilizados nessa pesquisa: Quadra de voleibol coberta com as marcações oficiais, fita $\operatorname{Scotch}{ }^{\circledR}$ $50 \mathrm{~mm}$ x 30m, rede de voleibol oficial fixada com cabos de aço nos postes, trena metálica de 10 metros 33 FT SEGA®, hardware, (dois sensores de piso com laser, interface, cabos e conectores), software específico, microcomputador Toshiba Satellite ${ }^{\circledR}$ e mesa suporte para microcomputador.

\section{PROCEDIMENTOS PARA A COLETA DE DADOS}

$A$ coleta de dados procedeu-se iniciando pela convocação de um estudante de Educação Física, atleta de voleibol, para o treinamento do manuseio do equipamento no que se refere à montagem e ao funcionamento do instrumento de medição para a aplicação, durante todo o período de ajustes e reajustes do mesmo até a sua calibração final, bem como para o entendimento dos movimentos específicos analisados. Assim, foram determinados dois avaliadores: o pesquisador e o estudante. Após essa fase, realizou-se contato com os dirigentes dos clubes e técnicos das equipes responsáveis para que os objetivos da pesquisa e a necessidade da participação dos mesmos fossem apresentados e, paralelamente, entregou-se o formulário do comitê de ética para que fosse assinado. 
Em seguida, procedeu-se da mesma forma com os atletas e seus pais, entregando-se, a cada um dos envolvidos, o termo de consentimento livre e esclarecido, de acordo com o Ministério da Saúde, resolução 196/96. Logo após os consentimentos e o projeto devidamente aprovado pelo Comitê de Ética em Pesquisa da Faculdade de Ciências Médicas da Universidade Estadual de Campinas processo 01/2005, deu-se início ao procedimento de coleta, conforme o agendamento com os técnicos e atletas.

Os testes foram realizados no mês julho de 2004 nas dependências da AABB (Associação Atlética do Banco do Brasil), na Cidade de Campina Grande PB. Procedeu-se com a coleta de dados utilizando-se do hardware e software validado por Sousa e Pellegrinotti (2005) em "Validade de equipamento eletrônico informatizado para análise de movimentos técnicos do voleibol: um estudo na categoria juvenil".

O teste foi composto por cinco bloqueios, na seguinte seqüência: saída da posição 3 deslocando-se lateralmente até a posição 2 , salto com os pés unidos, tanto na fase de impulsão, quanto na queda, executando o gesto técnico do bloqueio, voltando em seguida para posição inicial. Antes do início dos testes, o atleta executou o salto de forma lenta, apenas simulando os gestos para familiarizar-se com as ações e com o equipamento, pronto, apenas aguardando o avaliador para acionar o botão de comando do início do teste.

Durante a execução dos testes, os indivíduos realizaram uma seqüência de cinco bloqueios com 15 segundos de intervalo na zona de salto na posição 3 , centro da rede marcada com fita adesiva. Para iniciar, o atleta após um sinal sonoro (bip), saltou o mais alto possível, seguido por 15 segundos de repouso, finalizando pelo sinal sonoro emitido pelo computador. Após cada repetição desse ciclo de deslocamento lateral e salto, houve uma contínua sinalização de bips para comunicar o final do repouso e o reinício de mais um salto, após três segundos. $\mathrm{O}$ atleta imediatamente posicionou-se no ponto inicial e esperou o som de um bip prolongado e único, iniciando nova repetição, até completar cinco vezes essa mesma ação.

Após 24 horas, foi feito o reteste com o mesmo avaliador, nos mesmos atletas e nas mesmas condições. $\mathrm{Na}$ semana seguinte, com sete dias de intervalo, o segundo avaliador realizou o mesmo procedimento, utilizando os mesmos atletas e condições do teste e reteste, repetindo e conservando esse procedimento, $\mathrm{o}$ que totalizou 15 dias. 


\section{PLANO ANALÍTICO}

No plano de análise dos dados, utilizou-se o pacote estatístico Statiscal Package for Science Social (SPSS) 13.0 na criação e análise do banco de dados. Para a estatística descritiva, foram obtidos a média e coeficiente de variação (CV). Para a inferencial recorreu-se ao teste de Alpha de Cronbach, coeficiente de correlação "r" de Pearson, Coeficiente de Correlação Intraclasse (CCI), com nível de significância de $5 \%$ e intervalos com $95 \%$ de confiança para repetibilidade e reprodutibilidade.

\section{RESULTADOS}

Dois avaliadores T1 eT2 realizaram cinco medidas repetidas da altura do salto vertical em 18 atletas cujosresultados estão apresentados na Tabela 1.

\section{Tabela 1: Medida de cinco repetições daaltura do salto vertical em 18 atletas realizadas pelos avaliadores $\mathrm{T} 1 \mathrm{e} T 2$.}

\begin{tabular}{|c|c|c|c|c|c|c|c|c|c|c|c|c|}
\hline \multirow{3}{*}{ Atleta } & \multicolumn{6}{|c|}{$\mathrm{Tl}$} & \multicolumn{6}{|c|}{$\mathrm{T} 2$} \\
\hline & \multicolumn{5}{|c|}{ Repetiçá̃o } & \multirow[t]{2}{*}{$\bar{I}$} & \multicolumn{5}{|c|}{ Repetiçáo } & $\bar{X}$ \\
\hline & 1 & 2 & 3 & 4 & 5 & & 1 & 2 & 3 & 4 & 5 & \\
\hline 1 & 45 & 43 & 43 & 43 & 43 & 44,0 & 45 & 43 & 45 & 45 & 42 & 43,5 \\
\hline 2 & 45 & 40 & 45 & 40 & 42 & 43,5 & 40 & 40 & 40 & 43 & 40 & 40,0 \\
\hline 3 & 45 & 48 & 45 & 48 & 46 & 45,5 & 48 & 45 & 40 & 43 & 43 & 45,5 \\
\hline 4 & 36 & 36 & 39 & 34 & 34 & 35,0 & 38 & 38 & 36 & 36 & 35 & 36,5 \\
\hline 5 & 36 & 34 & 35 & 31 & 31 & 33,5 & 30 & 35 & 31 & 33 & 30 & 30,0 \\
\hline 6 & 42 & 49 & 38 & 36 & 39 & 40,5 & 38 & 35 & 34 & 35 & 35 & 36,5 \\
\hline 7 & 35 & 35 & 33 & 33 & 33 & 34,0 & 34 & 34 & 34 & 31 & 31 & 32,5 \\
\hline 8 & 38 & 38 & 33 & 38 & 39 & 38,5 & 38 & 38 & 36 & 39 & 36 & 37,0 \\
\hline 9 & 39 & 42 & 35 & 38 & 38 & 38,5 & 40 & 40 & 40 & 38 & 36 & 38,0 \\
\hline 10 & 38 & 39 & 38 & 36 & 35 & 36,5 & 36 & 36 & 38 & 43 & 39 & 37,5 \\
\hline 11 & 31 & 27 & 27 & 29 & 29 & 30,0 & 31 & 30 & 29 & 30 & 31 & 31,0 \\
\hline 12 & 22 & 22 & 22 & 21 & 19 & 20,5 & 22 & 24 & 23 & 22 & 24 & 23,0 \\
\hline 13 & 35 & 36 & 39 & 35 & 40 & 37,5 & 34 & 36 & 34 & 43 & 43 & 38,5 \\
\hline 14 & 40 & 40 & 39 & 40 & 39 & 39,5 & 44 & 42 & 42 & 40 & 40 & 42,0 \\
\hline 14 & 40 & 40 & 39 & 40 & 39 & 39,5 & 44 & 42 & 42 & 40 & 40 & 42,0 \\
\hline 15 & 42 & 43 & 43. & 40 & 40 & 41,0 & 40 & 43 & 43 & 42 & 39 & 39,5 \\
\hline 16 & 43 & 42 & 43 & 45 & 40 & 41,5 & 42 & 45 & 46 & 45 & 46 & 44,0 \\
\hline 17 & 38 & 42 & 39 & 40 & 40 & 39,0 & 40 & 43 & 36 & 39 & 39 & 39,5 \\
\hline 18 & 33 & 33 & 33 & 33 & 34 & 33,5 & 34 & 33 & 33 & 31 & 33 & 33,5 \\
\hline
\end{tabular}


Quando se mede parâmetros como carga, velocidade de deslocamento, altura dos saltos verticais e/ou a distância percorrida em uma atividade física estamos tentando dimensionar de alguma forma o trabalho. Em algumas atividades físicas, como os desportos com bola, a tarefa de dimensionar o trabalho é complexa e esta acompanhada de diversas limitações. O voleibol, de certo modo possibilita dimensionar isoladamente suas principais habilidades, pela clareza que é definido o início, meio e fim da execução de algumas das habilidades (saltos verticais, pequenos deslocamentos, mudanças de direção, gestos técnicos do ataque e saques, velocidade da bola no ataque, velocidade da bola no saque, entre outras).

De acordo com o Gráfico 1, a equação de regressão estimada para a altura do salto médio de T1 (=y) e T2 (=x) estão muito próximas da função identidade $\mathrm{y}=\mathrm{x}$. Isto mostra que as medidas apresentam boa repetibilidade. Numa escala de 0 a 1 , pode-se afirmar que esta repetibilidade é a correlação entre as médias de $\mathrm{T} 1$ e as médias de T2, igual a $0,9310$.

\section{Gráfico 1 - Grau de repetibilidade da altura do salto média para os avaliadores T1 e T2.}

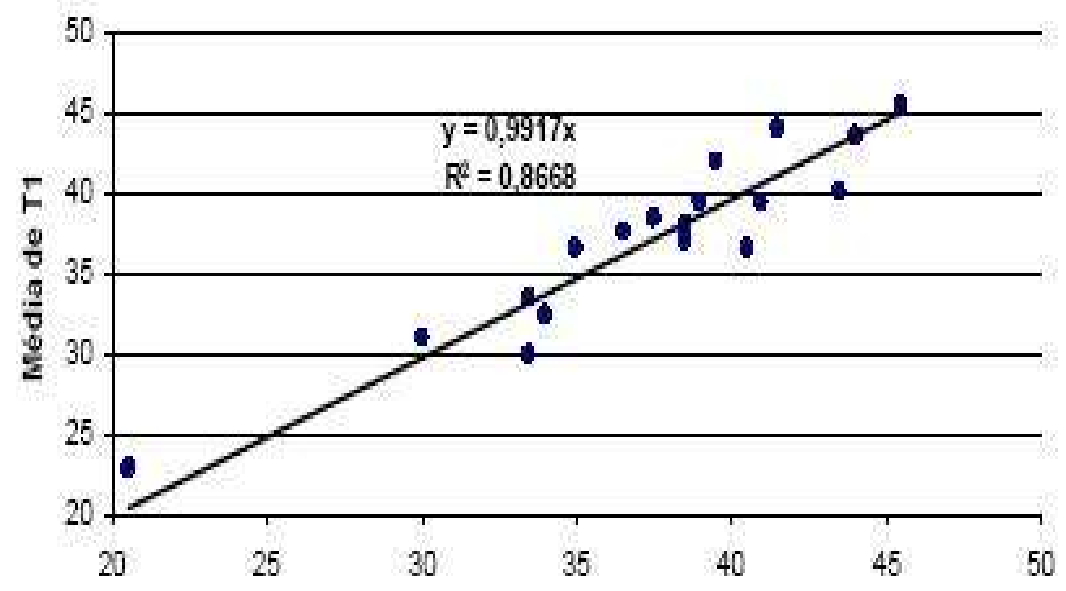

Média de T2

Um processo de medição é definido como consistente se o gráfico de controle da amplitude construído para medidas repetidas de um mesmo item indicar um estado de controle estatístico (WERKEMA, 1996). Pode-se observar que nenhuma amplitude observada está fora de controle, atestando a consistência das medições realizadas com o instrumento em estudo é consistente. Portanto, o Gráfico 2 mostra que o processo de medição realizado 
Gráfico 2 - Carta de controle para a Amplitude |( média de T1 - média de T2).

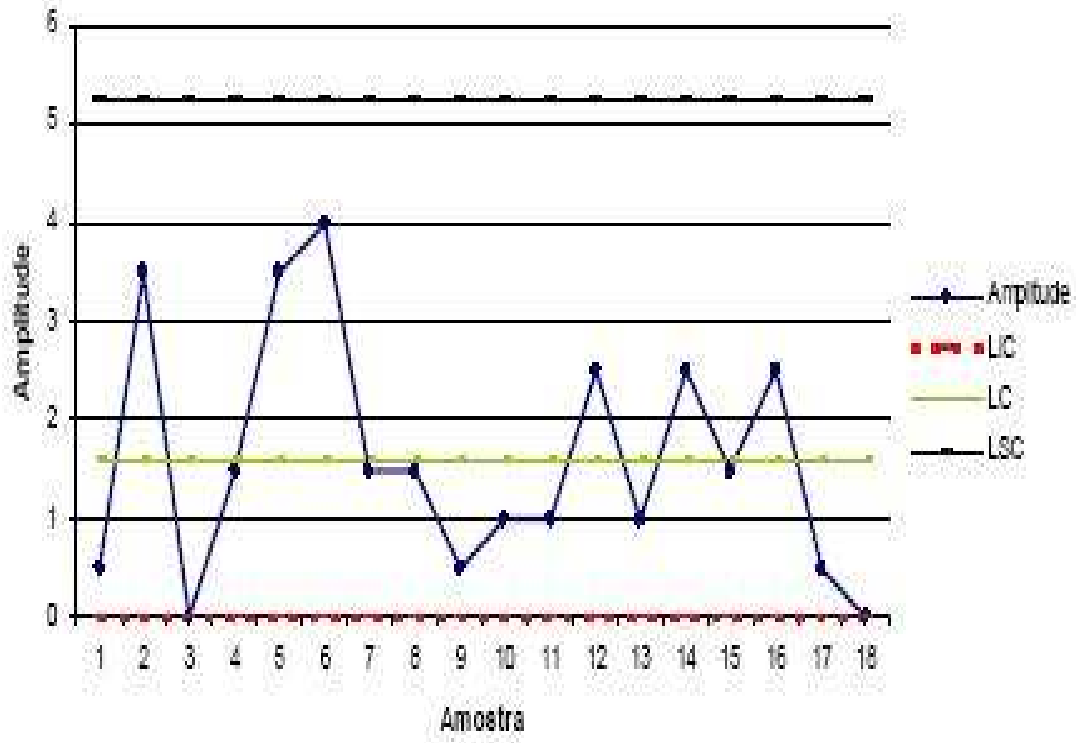

Pode-se observar na Tabela 2 que a validação do instrumento é confirmada com as medidas apresentadas.

Tabela 2 - Validação do instrumento: Repetibilidade pela medida de fidedignidade Alpha de Cronbach, coeficiente de correlação intraclasse, correlação de Pearson e coeficiente de variação.

\begin{tabular}{lcc} 
Comparação das medidas de T1 e T2 & Estatística & IC com 95\% \\
\hline Alpha de Cronbach & 0,9669 & 0,9116 a 0,9876 \\
$\begin{array}{l}\text { Coeficiente de correlação } \\
\text { intraclasse }\end{array}$ & 0,9360 & 0,8376 a 0,9756 \\
Correlação de Pearson & 0,9310 & - \\
Coeficiente de variação (\%) & 15,14 & - \\
\hline
\end{tabular}

A vantagem de apresentar a repetibilidade e a reprodutibilidade na forma da Tabela 2, ou seja, apresentando uma estimativa pontual e intervalar para seus valores baseado no conceito probabilístico da mesma que interpreta a reprodutibilidade e repetibilidade respectivamente como um percentil das variações nas medidas e nas médias (HAMAOKA; SILVA, 1998; HRADESKY, 1989) traz uma resposta CONEXÕES, revista da Faculdade de Educação Física da UNICAMP, Campinas, v. 6, ed. especial, p. 111- , jul. 2008. 
que os demais trabalhos não atendem. De modo que se obtém um critério para decidir quando duas medições de um mesmo indivíduo realizado por um operador é aceitável ou quando duas medições de atletas distintos é considerada dentro de limites aceitáveis. Este critério produz uma orientação clara para os avaliadores que até então se baseiam em informações subjetivas do tipo baixo coeficiente de variação e equação de regressão próximas em medidas repetidas (LOTHIAN, FARRALLY; MAHONEY, 1993) em elevada correlação intraclasse, correlação de Pearson e ANOVA com dois fatores para medidas repetidas com o associado teste de Tukey para detectar tratamentos homogêneos (MELANSON et al., 1996); ANOVA com uma classificação e intervalos Média, Desvio padrão e gráfico de médias (MCLAUGHLIN et al., 2001); Média \pm desvio padrão, Coeficiente de variação, Teste t-Student, Coeficiente de Correlação de Pearson.

$\mathrm{Na}$ literatura científica, a repetibilidade e reprodutibilidade nas Ciências do esporte não utiliza as técnicas de Controle Estatístico de Qualidade e a estimativa pontual e por intervalo para a repetibilidade e reprodutibilidade, enquanto que na área de Engenharia de Qualidade esta forma de calcular a repetibilidade e reprodutibilidade são comuns.

A maior dificuldade observada nos executantes foi em coordenar essas passadas e seguir para a fase de vôo dentro dos limites dos sensores que registram esses gestos. Essa analise nos faz perceber que quanto mais imaturo tecnicamente for o indivíduo mais erros ele poderá cometer durante a execução dos testes.

Na maioria dos problemas de validação de instrumento temos o mesmo problema de engenharia, porém de forma mais complexa, pois peças não se deformam quando mudam de avaliadores e atletas podem apresentar performances variadas em momentos distintos. $\mathrm{Na}$ área de Engenharia o problema da especificação para a qualidade tolerável do produto é natural, porém ainda não temos uma especificação para a qualidade do atleta. Por esta razão tornou-se inviável determinar o percentual de tolerância do instrumento.

A quantificação dos saltos reforça as pesquisas já existentes e com isso justifica a sua utilização. Os estudos de Lacomi, Melis e Crisafulli (1998) corroboram com o nosso quando afirmam que é importante quantificar o salto vertical do jogador de voleibol.

\section{CONCLUSÕES}

Entre os avaliadores as medidas se apresentam com reprodutibilidade e repetibilidade favoráveis ao 
estudo de validação da plataforma de salto. Além disso, o processo de medição do teste de salto vertical está dentro do controle exigido por técnicas estatísticas de qualidade para indicar a validade por meio da reprodutibilidade. Ainda que, todas as correlações do estudo estão acima de 0,60 e os ICC de 0,90, indicando que o processo de validação é pertinente. Neste estudo a proposta de validação é aceitável e satisfatória com base e de conformidade com os índices da literatura.

\section{REFERÊNCIAS}

ARTEAGA, R; et al. Reliability of jumping performance in active men and women under different stretch loading conditions. J Sports Med Phys Fitness, v. 40, n. 1, p. 26-34, 2000.

ATKINSON, G.; NEVILL, A M. Statistical methods for assessing measurement error (reliability) in variables relevant to sports medicine. Sports Med, v. 26, n. 4, p. 217-238.

CRONIN, J. B; HING, R. D; MCNAIR, P. J. Reliability and validity of a linear position transducer for measuring jump performance. J Strength Cond Res, v. 18, n. 3, p. 590-593, 2006.

CRONIN, J. B.; HANSEN, K T. Strength and power predictors of sports speed. J Strength Cond Res 2005, v. 19, n. 2, p. 349-357, 2005.

HAMAOKA, R. E.; SILVA, P. A. L. Repetibilidade e reprodutibilidade. Relatório Técnico $n$. 030/dez./98. Rio de Janeiro: Instituto Militar de Engenharia, 1998.

HOPKINS, W. G. Measures of reliability in sports medicine and science. Sports Méd, v. 30, n. 1, p. 115,2000 .

HRADESKY, J. L. Aperfeiçoamento da qualidade e da produtividade: guia prático para a implementação do controle estatístico de processo. Rio de Janeiro: McGraw-Hill, do Brasil, 1989.

LACONI, P.; MELIS, F.; CRISAFULLI, A. Field test for mechanical efficiency evaluation in mathing volleyball players. Int J Sports Med, v. 19, n. 1, p. 52-55, 1998.

LOTHIAN, F.; FARRALlY, M. R.; MAHONEY, C. Validity and Reliability of the K2 to Measure Oxygen Uptake. Can J Appl Physiol, v. 18, n. 2, p. 197-206, 1996.

MClAughlin, J. E.; KING, G. A; HOWLEY, E. T; BASSETT, D. R.; AINSWORTH, B. E. Validation of the COSMED K4 b2 Portable Methabolic System. Int J Sports Med, v. 22, p. 280-281, 2001.

MELANSON, E. L.; et al. Reliability and validity of a portable methabolic measurement system. Can J Appl Physiol, v. 21, 1996.

PLATONOV, V. N. Teoria geral do treinamento desportivo olímpico. Porto Alegre: Artmed, 2004.

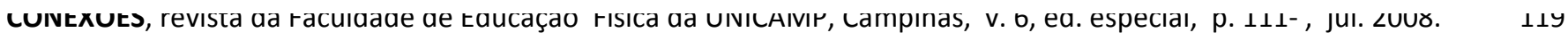
ISSN: $1983-9030$. 
SOUSA, S. J. G; PELLEGRINOTTI, I. L. TW20 metros: proposta computadorizada para análise da performance específica em atletas de voleibol, 2000. 113f. Dissertação (Mestrado)- Faculdade de Educação Física, Universidade Estadual de Campinas, Campinas, 2000.

SOUSA, S. J. G.; PELLEGRINOTTI, I. L. Validade de equipamento eletrônico informatizado para análise de movimentos técnicos do voleibol: um estudo na categoria juvenil. 2005. 159f. Tese (Doutorado) - Faculdade de Educação Física, Universidade Estadual de Campinas, Campinas, 2005.

WERKEMA, M. C. C. Avaliação das qualidades de medidas. Belo Horizonte: Fundação Christiano Ottoni, 1996. 


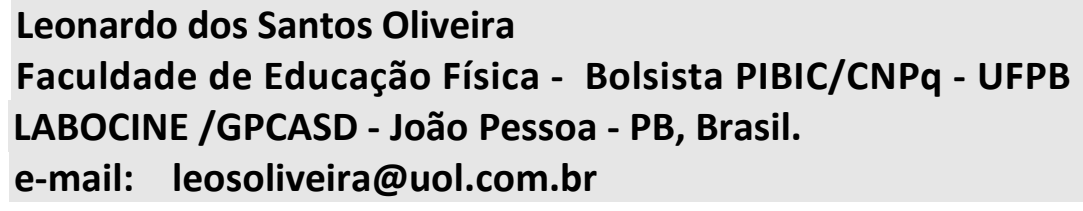

Solon José Gonçalves de Sousa

Universidade Federal da Paraíba

\section{Richard Valadares Silva}

Universidade Federal da Paraíba - LABOCINE /GPCASD

\section{Pedro lanaí Pordeus Uchôa \\ Universidade Federal das Paraíba - LABOCINE}

\section{Péricles Paes Barreto Correia}

Universidade Federal da Paraíba - LAAFISC

\section{Gilmário Ricarte Batista}

Universidade Federal do Rio Grande do Norte

\section{Idico Luiz Pellegrinotti}

Universidade Metodista de Piracicaba

Referência do artigo:

\section{ABNT}

OLIVEIRA, L. S., et al. Validação e reprodutibilidade de plataforma de salto com laser para altura do salto em voleibolistas. Conexões, v. 6, ed. especial, p. 111-121. 2008.

\section{APA}

Oliveira, L. S., Sousa S. J. G. , Silva R. V., Uchôa P. I. P., Correia P. P. B., Batista G. R., \& Pellegrinotti I. L. (2008). Validação e reprodutibilidade de plataforma de salto com laser para altura do salto em voleibolistas. Conexões, 6(ed. especial), 111-121.

\section{VANCOUVER}

Oliveira LS, Sousa SJG, Silva RV, Uchôa PIP, Correia PPB, Batista GR, Pellegrinotti IL. Validação e reprodutibilidade de plataforma de salto com laser para altura do salto em voleibolistas. Conexões, 2008; 6(ed. especial): 11-121. 